\title{
How many bits does it take to track an open quantum system?
}

\author{
R. I. Karasik - and H. M. Wiseman才 \\ Centre for Quantum Computer Technology, Centre for Quantum Dynamics, \\ Griffith University, Brisbane, Queensland 4111, Australia
}

\begin{abstract}
A $D$-dimensional Markovian open quantum system will undergo quantum jumps between pure states, if we can monitor the bath to which it is coupled with sufficient precision. In general these jumps, plus the between-jump evolution, create a trajectory which passes through infinitely many different pure states. Here we show that, for any ergodic master equation, one can expect to find an adaptive monitoring scheme on the bath that can confine the system state to jumping between only $K$ states, for some $K \geq(D-1)^{2}+1$. For $D=2$ we explicitly construct a 2 -state ensemble for any ergodic master equation, showing that one bit is always sufficient to track a qubit.
\end{abstract}

PACS numbers: 03.65.Yz, 03.65.Aa, 42.50.Lc, 42.50.Dv

The first quantitative model of quantum dynamics was Einstein's model of stimulated and spontaneous jumps [1] between Bohr's stationary atomic states [2]. In modern language, this is a model for an open quantum system weakly coupled to a heat bath, and, as Einstein showed, such jumps can lead to an equilibrium state that is a thermal mixture of energy eigenstates, with finite entropy. In Einstein's model, if one could track the individual stochastic events of energy exchange between atom and bath, then one would know which energy eigenstate the system occupied at any time. Truncating to a finite number $D$ of energy eigenstates, it follows that only a finite classical memory is required to keep track of the quantum system (that is, to know its exact pure state) in thermal equilibrium: a $K$-state memory with $K=D$.

Einstein's theory is a special case of Markovian open quantum system dynamics for finite-dimensional systems, which most generally are describable by a Lindblad-form master equation (ME) [3] :

$$
\dot{\rho}=\mathcal{L} \rho \equiv-i\left[\hat{H}_{\mathrm{eff}} \rho-\rho \hat{H}_{\mathrm{eff}}^{\dagger}\right]+\sum_{l=1}^{L} \hat{c}_{l} \rho \hat{c}_{l}^{\dagger},
$$

where $\hat{H}_{\text {eff }} \equiv \hat{H}-i \sum_{l} \hat{c}_{l}^{\dagger} \hat{c}_{l} / 2$. Here $\hat{H}$ is Hermitian (it is the Hamiltonian) but the jump operators $\left\{\hat{c}_{l}\right\}$ are completely arbitrary. Einstein's theory is a special case because in it each jump operator is proportional to $|E\rangle\left\langle E^{\prime}\right|$, for some $\hat{H}$-eigenstates $|E\rangle$ and $\left|E^{\prime}\right\rangle$, so that the state after any jump is a stationary state $|E\rangle$. For a general $\mathrm{ME}$, it is always possible, in principle, to monitor the bath such that every jump is resolvable, so that the system can be known to be in some pure state $|\psi(t)\rangle$ at all times [3-5]. However, in general, after a jump at time $\tau_{j}$, the state $\propto \hat{c}_{l}\left|\psi\left(\tau_{j}\right)\right\rangle$ will depend on the prejump state $\left|\psi\left(\tau_{j}\right)\right\rangle$, and will not be an an eigenstate of $\hat{H}$. Even if it were an energy eigenstate, it would not in general remain stationary until the next jump, because its subsequent evolution would be generated by the effective (non-Hermitian) Hamiltonian $\hat{H}_{\text {eff }}$ appearing in Eq. (1).

It is thus not at all obvious whether for a general finite- dimensional open quantum system it would be possible to keep track of its pure state, even in principle, with a finite classical memory. On the face of it, it would seem necessary to store the exact times of each jump - a sequence of real numbers $\left\{\tau_{j}: j\right\}$ each of which would require, in principle, an infinite memory to store. Alternately one could store the conditioned quantum state $|\psi(t)\rangle$ itself, but this (a $D$-dimensional complex vector) would also require an infinite memory. This situation is of course completely different from a finite-state stochastic classical system (which is what Einstein's model amounts to), where a finite-state classical memory of the same size is always sufficient.

In this Letter we address this fundamental question about open quantum system dynamics. We show that for any ergodic Markovian dynamics 15] of a $D$-dimensional quantum system, one can expect to be able to track the state with a $K$-state classical apparatus for some $K \geq(D-1)^{2}+1$. This is possible only because there is entanglement between the system and bath, which means that different monitoring schemes on the bath give rise to different sorts of stochastic pure state trajectories ("unravellings" [4]) for a given $\mathrm{ME}$. We then prove that for $D=2$ (a qubit), $K=2$ is always sufficient; that is, there is always an unravelling for which the qubit jumps between only two possible states, $\left|\phi_{1}\right\rangle$ and $\left|\phi_{2}\right\rangle$. Although this sounds similar to Einstein's dynamics, it is in fact quite different in general - the two states are non-orthogonal, $\left\langle\phi_{1} \mid \phi_{2}\right\rangle \neq 0$, and the monitoring of the qubit's environment must be adaptive, controlled by the classical bit that stores the state of the qubit.

We begin by revisiting the preferred ensemble fact [6] , to explain why it is not possible in general to unravel a ME such that the system jumps between the eigenstates of the equilibrium $\rho$ (as in Einstein's model). Then we show the general result cited above for $D$-dimensional systems, and give an explicit construction of the adaptive unravelling for the special case of cyclic jumps with only one Lindblad operator $\hat{c}$. We then prove that one bit is always sufficient to track a qubit, and that in some cases it is actually possible to store the state of the open 
qubit using less than one bit of memory on average, if we imagine an ensemble of $N \gg 1$ qubits, each independently measured. Surprisingly, considering $K>2$ can actually help in this regard. We illustrate these phenomena using the resonance fluorescence ME [4].

The preferred ensemble fact. - Consider a Linbladian $\mathcal{L}$ with unique steady state defined by $\mathcal{L} \rho_{\mathrm{ss}}=0$ and we assume that $\rho_{\mathrm{ss}}$ is a mixed state. This mixed state can be decomposed in terms of pure states $\left|\phi_{k}\right\rangle$ via $\rho_{\mathrm{ss}}=\sum_{k=1}^{K} \wp_{k}\left|\phi_{k}\right\rangle\left\langle\phi_{k}\right|$ with positive constants $\wp_{k}$. Note that there are infinitely many such decompositions, as the states $\left|\phi_{k}\right\rangle$ need not be orthogonal. However, only for some decompositions is it possible to devise a way to monitor the system's environment - which leaves the average evolution of the system unchanged from Eq. (1) - such that the system will only ever be in one of the states $\left|\phi_{k}\right\rangle$, and will spend a proportion of time in that state equal to $\wp_{k}$ in the long-time limit. Decompositions $\left\{\wp_{k},\left|\phi_{k}\right\rangle\right\}$ that can be realized in this way are called physically realizable $(\mathrm{PR})$. The fact that some decompositions are not $\mathrm{PR}$ is known as the preferred ensemble fact [6].

As shown in Ref. [6], an ensemble $\left\{\wp_{k},\left|\phi_{k}\right\rangle\right\}$ is PR iff (if and only if) there exists rates $\kappa_{j k} \geq 0$ such that

$$
\forall k, \mathcal{L}\left|\phi_{k}\right\rangle\left\langle\phi_{k}\right|=\sum_{k=1}^{K} \kappa_{j k}\left(\left|\phi_{j}\right\rangle\left\langle\phi_{j}|-| \phi_{k}\right\rangle\left\langle\phi_{k}\right|\right) .
$$

For a general ME, most decompositions $\left\{\wp_{k},\left|\phi_{k}\right\rangle\right\}$ of $\rho_{\mathrm{ss}}$ are not $\mathrm{PR}$, including the $K=D$ ensemble composed from the diagonal basis for $\rho_{\mathrm{ss}}[3]$.

The existence of $P R$ ensembles. - For finite $K$ and $D$, searching for solutions of Eq. (2) reduces to solving polynomial equations. We can describe $K$ pure states with $K(2 D-1)$ real unknowns and $K$ quadratic constraints from normalization. Eq. (2) introduces $K^{2}-K$ unknown rates $\kappa_{j k}$ and imposes an additional $K\left(D^{2}-1\right)$ cubic constraints (the minus one is because both sides are traceless by construction). Thus we have $K D^{2}$ polynomial constraints and $K(2 D+K-2)$ unknowns. For $K>(D-1)^{2}+1$ we have an underdetermined system of equations. For linear underdetermined systems, given by equations $\left\{f_{j}\right\}$, there are infinitely many solutions except for the set of measure zero for which there exist constants $\left\{\alpha_{j}\right\}$ such that $\sum_{j} \alpha_{j} f_{j}+1=0$. Similarly, for polynomial systems $\left\{p_{j}\right\}$, the Real Nullstellensatz [11] certifies that there are no real solutions iff there exist some polynomials $\left\{a_{j}\right\}$ and $\left\{d_{k}\right\}$ such that $\sum_{j} a_{j} p_{j}+\sum_{k} d_{k}^{2}+1=0$. Thus, for a general ME we expect to be able to find a $K$-element PR ensemble for some $K-1 \geq(D-1)^{2}$.

The freedom that experimentalists have (in principle) to realize different PR ensembles comes from the ability to monitor the system's environment in different ways. This can be understood as follows. The ME (11) is invariant under the transformations $\left\{\hat{c}_{l}\right\} \rightarrow\left\{\hat{c}_{m}^{\prime}\right\}$, where $\hat{c}_{m}^{\prime}=\sum_{l=1}^{L} S_{m l} \hat{c}_{l}+\beta_{m}$, and $\hat{H} \rightarrow \hat{H}^{\prime}=\hat{H}-$ $\frac{i}{2} \sum_{m=1}^{M}\left(\beta_{m}^{*} \hat{c}_{m}^{\prime}-\beta_{m} \hat{c}_{m}^{\prime}{ }^{\dagger}\right)$. Here $\vec{\beta}$ is an arbitrary complex vector and $\mathbf{S}$ is an arbitrary semi-unitary matrix $\sum_{m=1}^{M} S_{l^{\prime} m}^{*} S_{m l}=\delta_{l^{\prime}, l}$. Unravelling this ME with $\left\{\hat{c}_{m}^{\prime}\right\}$ as the jump operators and $\hat{H}_{\text {eff }}^{\prime}=\hat{H}^{\prime}-i \sum_{m=1}^{M} \hat{c}_{m}^{\prime}{ }^{\dagger} \hat{c}_{m}^{\prime} / 2$ as the effective non-Hermitian Hamiltonian clearly gives different stochastic evolution, while leaving the average evolution unchanged. To obtain the most general purestate unravelling of the ME, we require $\vec{\beta}$ and $\mathbf{S}$ to depend upon the previous record of jumps. That is, we require an adaptive monitoring [3, 5]. Of course when we use this to achieve jumping between a finite number of states, the classical $K$-state memory that stores which state the system is currently in carries all the information necessary for determining $\vec{\beta}$ and $\mathbf{S}$. That is, the adaptive unravelling is specified by $K$ different values for $\vec{\beta}$ and $\mathbf{S}$. The physical meaning of these parameters is most easily explained in a quantum optics context: $\mathbf{S}$ describes a linear interferometer [16] taking the field outputs from the system as inputs, while $\vec{\beta}$ describes adding (weak) local oscillators to the output fields from the interferometer prior to detection by photon counting. Recently, adaptive control of a weak local oscillator has been used for optimally distinguishing coherent states [8], and of a strong local oscillator for improved phase estimation [9].

Backing out the measurement scheme. — Although for every PR ensemble there must exist a monitoring scheme exists by definition, it may not be easy to find. We now present an explicit method for determining this scheme for the special case of cyclic jumps with a single Lindblad operator $\hat{c}$. That is, we assume that the system in the state $\left|\phi_{k}\right\rangle$ always jumps to the state $\left|\phi_{k+1}\right\rangle$ (strictly, $\left|\phi_{(k+1) \bmod K}\right\rangle$ ). In this case there are only $K$ jump rates, so the number of real unknowns is only $2 K D$. With a single Linblad operator and cyclic jumps, both sides of Eq. (2) have rank two by construction, so for $D>2$ Eq. (2) is less constraining than in the general case. Nevertheless, the system will be overconstrained for $D>2$. Thus in general we do not expect there to exist cyclic jump solutions for $D>2$. Later we exhibit eleven different cyclic jump solutions for a qubit $(D=2)$, and the method here is applicable to each of them.

In the case of a single Lindblad operator, the only freedom in the unravelling is in choosing $\beta^{k}$, the local oscillator amplitude when the system is known to be in state $\left|\phi_{k}\right\rangle$ (that is, when the $K$-state classical memory is in state $k$ ). This gives the jump operator $\hat{c}+\beta^{k}$ and the effective Hamiltonian $\hat{H}_{\text {eff }}^{k}=\hat{H}_{\text {eff }}+i \beta^{k *} \hat{c}-i\left|\beta^{k}\right|^{2} / 2$. Thus the system will undergo cyclic jumps iff

$$
\left(\hat{H}_{\mathrm{eff}}+i \beta^{k *} \hat{c}\right)\left|\phi_{k}\right\rangle \propto\left|\phi_{k}\right\rangle, \quad\left(\hat{c}+\beta^{k}\right)\left|\phi_{k}\right\rangle \propto\left|\phi_{k+1}\right\rangle,
$$

Now by assumption from Eq. (2), $\mathcal{L}\left|\phi_{k}\right\rangle\left\langle\phi_{k}\right| \propto$ $\left|\phi_{k+1}\right\rangle\left\langle\phi_{k+1}|-| \phi_{k}\right\rangle\left\langle\phi_{k}\right|$. From this we can show that

$$
\begin{aligned}
\hat{c}\left|\phi_{k}\right\rangle & =a_{k}\left|\phi_{k}\right\rangle+b_{k}\left|\phi_{k+1}\right\rangle, \\
\hat{H}_{\text {eff }}\left|\phi_{k}\right\rangle & =c_{k}\left|\phi_{k}\right\rangle+i a_{k}^{*} b_{k}\left|\phi_{k+1}\right\rangle .
\end{aligned}
$$


For some coefficients $a_{k}, b_{k}$ and $c_{k}$ that are easily found given $\left|\phi_{k}\right\rangle$ and $\left|\phi_{k+1}\right\rangle$. Comparing this to Eq. (3), we see that choosing $\beta^{k}=-a_{k}$ gives cyclic jumps as required.

For a qubit, one bit is all it takes. - We now prove that a 2-state PR ensemble always exists for a qubit. We use the Bloch representation, so that Eq. (1) becomes

$$
\dot{\vec{r}}=A \vec{r}+\vec{b},
$$

where $A$ is a $3 \times 3$-matrix and $\vec{b}$ is a 3 -vector. As always, we assume that there exists a unique steady state $\vec{r}_{\mathrm{ss}}=-A^{-1} \vec{b}$, which is the case iff the real part of each eigenvalue of $A$ is negative. We can track this system with a $K$-state memory iff there exists an ensemble $\left\{\wp_{k}, \vec{r}_{k}\right\}$ and rates $\kappa_{j k} \geq 0$ such that

$$
\begin{aligned}
\forall k, \vec{r}_{k} \cdot \vec{r}_{k} & =1, \\
\forall j, A \vec{r}_{j}+\vec{b} & =\sum_{k=1}^{K} \kappa_{j k}\left(\vec{r}_{k}-\vec{r}_{j}\right) .
\end{aligned}
$$

Thus the problem reduces to finding a real solution to a system of quadratic equations with real coefficients. This type of problem is surprisingly hard even for a small number of unknowns, and is known to be an NP-complete problem in general [10].

Luckily, the simplest case of $K=2$ has an analytical solution. Here the qubit is assumed to jump between two states, $\vec{r}_{1}$ and $\vec{r}_{2}$. Then Eqs. (8) reduce to a single equation, $A\left(\vec{r}_{1}-\vec{r}_{2}\right)=\left(\kappa_{12}+\kappa_{21}\right)\left(\vec{r}_{2}-\vec{r}_{1}\right)$, which is simply an eigenvalue equation. Thus $\vec{r}_{1}-\vec{r}_{2}=\vec{v}$ is an eigenvector of $A$ with eigenvalue $\lambda=-\left(\kappa_{12}+\kappa_{21}\right)$. From Eq. (7) and the fact that $\wp_{1}=\kappa_{21} /\left(\kappa_{21}+\kappa_{12}\right)=1-\wp_{2}$, it is simple to show that $\vec{r}_{1}$ and $\vec{r}_{2}$ have the form

$$
\begin{aligned}
& \vec{r}_{1}=\vec{r}_{\mathrm{sS}}+\check{v} \sqrt{1-\left\|\vec{r}_{\mathrm{sS}}\right\|^{2}} \sqrt{\wp_{1} /\left(1-\wp_{1}\right)} \\
& \vec{r}_{2}=\vec{r}_{\mathrm{sS}}-\check{v} \sqrt{1-\left\|\vec{r}_{\mathrm{sS}}\right\|^{2}} \sqrt{\left(1-\wp_{1}\right) / \wp_{1}}
\end{aligned}
$$

with $\check{v}=\vec{v} /\|\vec{v}\|$ is the normalized eigenvector, and

$$
\wp_{1}=\frac{1}{2}\left(1-\frac{\left\langle\vec{r}_{\mathrm{ss}}, \check{v}\right\rangle}{\sqrt{1-\left\|\vec{r}_{\mathrm{sS}}\right\|^{2}+\left\langle\vec{r}_{\mathrm{ss}}, \check{v}\right\rangle^{2}}}\right)=\frac{\kappa_{21}}{|\lambda|} .
$$

Because the Bloch vectors must be real, only real eigenvectors $\vec{v}$ of $A$ can contribute to the solution. By assumption, $A$ has three nonzero eigenvalues and, by the fundamental theorem of algebra, at least one eigenvalue (and consequently one eigenvector) is real. Therefore, a qubit always has a preferred ensemble comprising just two states.

Entropy. - As nooted above, it may be possible to store the state of a qubit in less than one bit, in an average sense. We can quantify this by using the Shannon entropy. Under continuous monitoring, in the longtime limit, the system will occupy states $\left|\phi_{k}\right\rangle$ with probabilities $\wp_{k}$. The Shannon entropy for this ensemble is $h\left(\left\{\wp_{k}\right\}\right)=-\sum_{k} \wp_{k} \log _{2} \wp_{k}$. This is lower bounded by the von Neumann entropy for the steady-state mixture:

$$
h\left(\left\{\wp_{k}\right\}\right) \geq S\left(\rho_{\mathrm{ss}}\right) \equiv-\operatorname{Tr}\left[\rho_{\mathrm{ss}} \log _{2} \rho_{\mathrm{ss}}\right],
$$

with equality iff $\left\{\wp_{k},\left|\phi_{k}\right\rangle\left\langle\phi_{k}\right|\right\}$ is the diagonal ensemble.

Note that if the eigenvector $\vec{v}$ of matrix $A$ used to construct the ensemble in Eqs. (9) 10] is orthogonal to the steady state $\vec{r}_{\mathrm{ss}}$, then the probability of occupying states $\vec{r}_{1}$ and $\vec{r}_{2}$ is $1 / 2$. In this case the Shannon entropy is 1 , meaning that one bit is sufficient to track the state of the system. If, on the other hand, $\vec{v}$ is not orthogonal to $\vec{r}_{\mathrm{ss}}$, then the Shannon entropy $h$ for the ensemble will be less than one and one could store the state on the qubit in less than one bit on average. That is, one could keep track of the state of a collection of $N$ identically monitored qubits using only $N h$ bits, in the limit of large $N$.

Resonance fluorescence. - In order to illustrate our ideas, we consider the example of resonance fluoresence of a two-level atom (a qubit) with basis states $|0\rangle$ and $|1\rangle$. The atom is coupled to the continuum of electromagnetic radiation and so decays to $|0\rangle$ at rate $\gamma$. At the same time, it is driven by a classical field with Rabi frequency $\Omega$. The qubit evolution in the interaction frame is given by a $\mathrm{ME}$ of the form of Eq. (11) with $\hat{H}=\Omega(|0\rangle\langle 1|+| 1\rangle\langle 0|) / 2$ and one jump operator $\hat{c}=\sqrt{\gamma}|0\rangle\langle 1|[3]$. For this case, $A$ and $\vec{b}$ in the Bloch vector equation, Eq. (6), are

$$
A=\left(\begin{array}{ccc}
-\gamma / 2 & 0 & 0 \\
0 & -\gamma / 2 & -\Omega \\
0 & \Omega & -\gamma
\end{array}\right) \quad \text { and } \vec{b}=\left(\begin{array}{l}
0 \\
0 \\
\gamma
\end{array}\right) \text {. }
$$

The steady state $\vec{r}_{\mathrm{ss}}=\left(0,2 \gamma \Omega,-\gamma^{2}\right)^{T} /\left(\gamma^{2}+2 \Omega^{2}\right)$, is a mixed state for $\Omega \neq 0$. The eigenvectors of $A$ are $\vec{v}_{1}=(1,00)^{T}$ and $\vec{v}_{ \pm}=\left(0, \gamma \pm \sqrt{\gamma^{2}-16 \Omega^{2}}, 4 \Omega\right)^{T}$. Eigenvector $\vec{v}_{1}$ is real and orthogonal to $\vec{r}_{\mathrm{ss}}$. Thus it will always yield a solution with Shannon entropy $h=1$. This solution was originally discovered in Ref. [7]. Eigenvectors $\vec{v}_{ \pm}$are real only for $|\Omega|<\gamma / 4$ and yield solutions with $h<1$. In fact, for $\epsilon \equiv \Omega^{2} / \gamma^{2} \ll 1$, the entropy of the solution due to $\vec{v}_{-}$differs from $S\left(\rho_{\mathrm{ss}}\right)$ only at $O\left(\epsilon^{3}\right)$. Thus for small driving, this ensemble has an entropy very close to the bound (12). This is seen in Fig. 1, where dashed lines show $h$ for the three different 2 -state solutions.

For $\epsilon>0.0625$ (that is, $|\Omega|>\gamma / 4$ ) there are no lowentropy 2 -state solutions. Surprisingly, by increasing $K$, the number of states, from 2 to 3 , we regain a relatively low entropy solution for some range of $\epsilon>0.0625$. Recall that for 2-state jumping, we use one real eigenvector of $A$ to construct the PR ensemble, as the Bloch vectors must be co-linear with $\vec{r}_{\mathrm{ss}}$. For 3 -state jumping, the Bloch vectors must be coplanar (and not colinear) with $\vec{r}_{\mathrm{ss}}$, so we require two eigenvectors. When $A$ has complex eigenvectors, they come in conjugate pairs, and we can, for some values of $\Omega$, construct a PR ensembles with 3 real Bloch vectors using these conjugate pairs. 


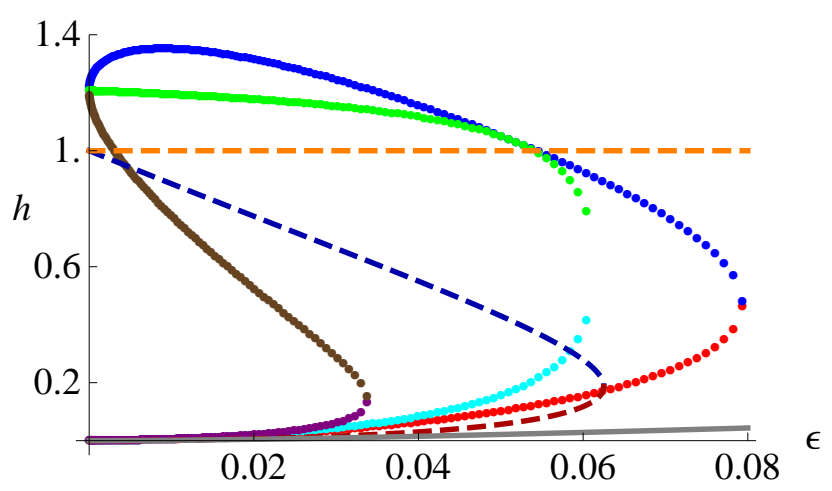

FIG. 1: The average number of bits required to keep track of the pure state of a qubit described by Eq. (13), as a function of dimensionless driving power $\epsilon=\Omega^{2} / \gamma^{2}$. The solid line is the von Neumann entropy for $\rho_{\mathrm{ss}}$, a lower bound on the memory required. The other nine curves are for eleven different adaptive unravellings. Dashed lines are for 2-state jumping. Dotted lines are for cyclic 3-state jumping.

We find all possible 3 -state cycles by numerical search for solutions to Eqs. (78) using symbolic-numerical algorithms based on computing Groebner bases [12]. There are eight solutions, coming in pairs, as shown (as dotted lines) in Fig. 1. For $\epsilon<0.0795$, there are two PR ensembles generated from complex eigenvectors $\vec{v}_{ \pm}$of $A$. These give the highest and lowest of the 3 -state jumping entropy curves. In the region $\epsilon<0.0610$, there are an additional four solutions constructed from $\vec{v}_{1}$ and $\vec{v}_{-}$. Only two new curves appear in Fig. 1 because they come in degenerate pairs. Finally, for $\epsilon<0.0335$, there are two more solutions constructed from $\vec{v}_{ \pm}$. As $|\Omega|$ decreases, the entropy for four of the solutions approaches 1.206, whereas the entropy for the other four approaches 0.

The three 2-state PR ensembles and two of the 3-state PR ensembles are shown in Fig. 2. Figure2(a) shows the main features of low-entropy solutions: the states are far apart, and the qubit spends most of the time in one state that is nearly aligned with the steady state. Figure 2(b) captures the nature of high-entropy solutions: the states cluster around steady state. In both of these cases the ensemble lies in the $x=0$ plane. The ensemble with $h=1$, Fig. 2(c) does not, but is still symmetric under reflection in this plane. This symmetry is respected for all ensembles found except for the pairs of 3-state jumping solutions that are degenerate with respect to entropy. Figure 2(d) shows one such pair: the two ensembles are mirror images of each-other in the $x=0$ plane.

In summary, we considered an arbitrary ergodic Markovian open quantum system subject to continuous monitoring that resolves every jump and allows the system to stay in a pure state. Under a generic monitoring scheme the system state will explore a manifold of pure states, so tracking it would require infinite memory. Here we showed that this situation is not an intrinsic property of open quantum systems, but is just a consequence
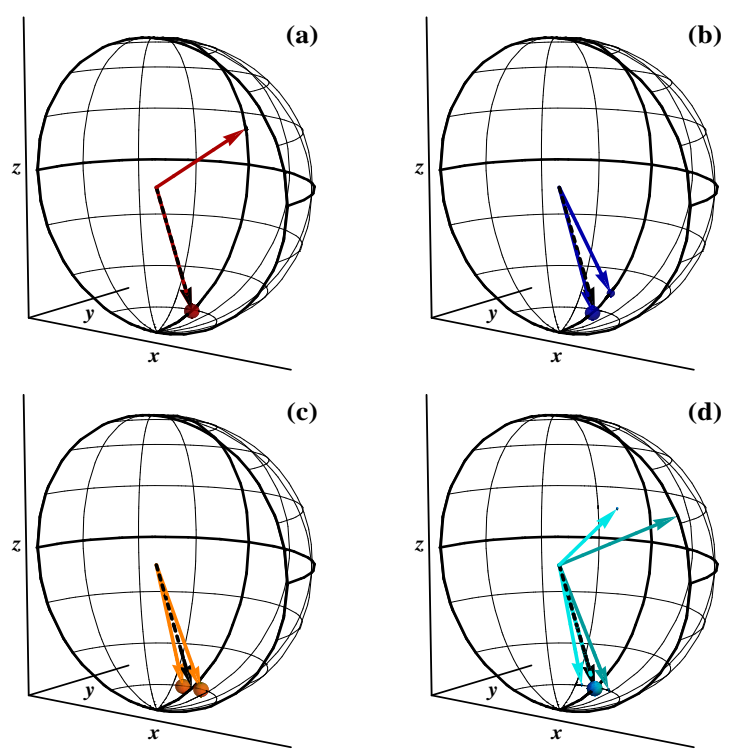

FIG. 2: Solid arrows show Bloch vectors for 2-state jumping (a)-(c), and 3-state jumping (d). Colors [online] match those of Fig. 1. The volume of the sphere at the tip of each arrow represents the probability that the qubit occupies the corresponding pure state. The dashed arrow is $\vec{r}_{\mathrm{ss}}$. For all plots, $\Omega / \gamma=0.2(\epsilon=0.04)$.

of using the "wrong" monitoring scheme - a finite $(K$ state) classical memory is sufficient to track the state of the system, by adaptively changing the scheme used to monitor the environment, controlled by the state of the classical memory that stores the state of the quantum system. In general one would expected to need at least $K=(D-1)^{2}+1$ classical states to track a $D$ dimensional quantum system. The gap between $K$ and $D$ may be related to the recent result that there are stochastic processes that can be generated using quantum systems of lower dimensionality than is possible using only classical systems [13]. The above quadratic difference, $K-1=(D-1)^{2}$, is also reminiscent of other comparisons between quantum and classical systems [14], so whether this $K$ is always sufficient is an important open question. For $D=2$, however, the answer is now known, and is: yes, one bit is always enough to track the state of a qubit.

This work was funded by the ARC grants CE0348250 and FF0458313.

* Electronic address: R.Karasik@Griffith.edu.au

† Electronic address: H.Wiseman@Griffith.edu.au

[1] A. Einstein, Phys. Zeits. 18, 121 (1917).

[2] N. Bohr, Phil. Mag. 26, 1 (1913).

[3] H. M. Wiseman and G. J. Milburn, Quantum Measurement and Control, (Cambridge University Press, 2010).

[4] H. J. Carmichael, An Open Systems Approach to Quantum Optics (Springer-Verlag, Berlin, 1993). 
[5] H. M. Wiseman, Quantum Semiclass. Opt 8, 205 (1996).

[6] H.M. Wiseman and J. A. Vaccaro, Phys. Rev. Lett. , 87, 240402 (2001).

[7] H. M. Wiseman and G. E. Toombes, Phys. Rev. A , 60, 2474 (1999).

[8] R. L. Cook, P. J. Martin, and J. M. Geremia, Nature 446, 774 (2007)

[9] T. A. Wheatley et al., Phys. Rev. Lett. 104, 093601 (2010).

[10] L. Blum, F. Cucker, M. Shub, and S. Smale, Complexity and Real Computation, (Springer-Verlag, 1997).

[11] B. Sturmfels, Solving Systems of Polynomial Equations
(CBMS Regional Conference Series in Mathematics), (American Mathematical Soceity, 2002).

[12] D.Cox, J. Little, and D. O'Shea, Ideals, Varieties, and Algorithms, (Springer-Verlag, 1997).

[13] A. Monras, A. Beige, and K. Wiesner, arXiv:1002.2337

[14] L. Hardy, arXiv:quant-ph/0101012

[15] Here ergodic simply means that the master equation has a unique stationary state.

[16] Here 'linear interferometer' is to be understood in the most general sense, including frequency shifters if the system has outputs in different frequency bands. 